\title{
Topological properties and fractal analysis of recurrence network constructed from fractional Brownian motions
}

\author{
Jin-Long $\mathrm{Liu}^{1}$, Zu-Guo $\mathrm{Yu}^{1,2 *}$ and Vo $\mathrm{Anh}^{2}$ \\ ${ }^{1}$ Hunan Key Laboratory for Computation and Simulation in Science and Engineering and \\ Key Laboratory of Intelligent Computing and Information Processing of Ministry of Education, \\ Xiangtan University, Xiangtan, Hunan 411105, China. \\ ${ }^{2}$ School of Mathematical Sciences, Queensland University of Technology, \\ GPO Box 2434, Brisbane, Q4001, Australia.
}

\begin{abstract}
Many studies have shown that we can gain additional information on time series by investigating their accompanying complex networks. In this work, we investigate the fundamental topological and fractal properties of recurrence networks constructed from fractional Brownian motions (FBMs). First, our results indicate that the constructed recurrence networks have exponential degree distributions; the average degree exponent $\langle\lambda\rangle$ increases first and then decreases with the increase of Hurst index $H$ of the associated FBMs; the relationship between $H$ and $\langle\lambda\rangle$ can be represented by a cubic polynomial function. We next focus on the motif rank distribution of recurrence networks, so that we can better understand networks at the local structure level. We find the interesting superfamily phenomenon, i.e. the recurrence networks with the same motif rank pattern being grouped into two superfamilies. Last, we numerically analyze the fractal and multifractal properties of recurrence networks. We find that the average fractal dimension $\left\langle d_{B}>\right.$ of recurrence networks decreases with the Hurst index $H$ of the associated FBMs, and their dependence approximately satisfies the linear formula $\left\langle d_{B}>\approx 2-H\right.$, which means that the fractal dimension of the associated recurrence network is close to that of the graph of the FBM. Moreover, our numerical results of multifractal analysis show that the multifractality exists in these recurrence networks, and the multifractality of these networks becomes stronger at first and then weaker when the Hurst index of the associated time series becomes larger from 0.4 to 0.95 . In particular, the recurrence network with the Hurst index $H=0.5$ possess the strongest multifractality. In addition, the dependence relationships of the average information dimension $\langle D(1)\rangle$ and the average correlation dimension $\langle D(2)\rangle$ on the Hurst index $H$ can also be fitted well with linear functions. Our results strongly suggest that the recurrence network inherits the basic characteristic and the fractal nature of the associated FBM series.
\end{abstract}

Key words: recurrence network; fractional Brownian motion; fractal dimension; multifractal analysis.

*Corresponding author, email: yuzg1970@yahoo.com 
PACS: $89.75 . H c, 05.45 . D f, 47.53 .+\mathrm{n}$

\section{Introduction}

Methods of nonlinear time series analysis have been widely applied in physics, physiology, finance and biology. Complex network theory has become one of the most important developments in statistical physics [1]. Recent studies have shown that complex network theory may be an effective method to extract the information embedded in time series $[2,3]$. Many complicated dynamics systems in nature and society can be described by complex networks. In a complex system, its elements are represented by nodes, and their interactions are represented by directed or undirected edges.

Based on the small-world and scale-free properties of complex networks [4,5], Newman et al. [6] investigated extensively the structure and function of real-world complex networks in different areas. The advancement of network theory provides us with a new perspective to perform time series analysis $[2,3]$. Therefore, we can further understand the structural features and dynamics mechanism of complex systems by studying the basic topological properties of networks. Many algorithms have been proposed to construct different complex networks from time series [7], such as visibility graphs $[8,9]$, space state networks [10], recurrence networks $[2,3,11]$, and nearest-neighbor networks $[12,13]$.

Recurrence is a basic characteristic of many complex dynamical systems, which can be used to describe the dynamics behavior of systems. Eckmann et al. [14] developed the method of recurrence plots (RPs) to visualize the recurrence property of complex dynamical systems. A remarkable advantage of this method is that it is very suitable for short and even non-stationary time series. Applications of the method of RPs can be found in various fields of research such as physiology, neuroscience, earth sciences, engineering, biology and finances. In order to quantify the structures of the recurrence plots, Zbilut and Webber developed the recurrence quantification analysis (RQA) based on linear and diagonal line structures of recurrence plots $[15,16]$. Most of these measures of complexity are available on TOCSY software platform [17]. Cross recurrence plot (CRP) [18,19] and joint recurrence plot (JRP) [20] are bivariate and multivariate extensions of the RPs, respectively.

Recently, Donner et al. [2,3] and Marwan et al. [11] proposed the recurrence network based on the method of RPs. This novel idea can be considered as a complementary view on the RQA, allowing us to gain additional information from corresponding network-theoretic measures which cannot be obtained by RQA. They considered the phase space state vectors $X(i)$ (defined in Section II) as nodes of a complex network and identify the recurrence matrix of the original time series with the adjacency matrix of an associated complex network. In this way, an undirected and unweighted network is represented by a binary adjacency matrix $A_{i, j}$ (defined in Section II). Recurrence networks have been used to many model systems such as logistic map, Hénon map, Lorenz system, Rössler system and Bernoulli map [3,11,21], and real economic series [22].

It is well known that fractional Brownian motion (FBM) described by the Hurst index $H(0<$ $H<1$ ) is a self-similar process with stationary increments [23]. The FBM with $H=1 / 2$ is in 
fact the classical Brownian motion. The correlation between increments of the FBM is negative if $0<H<1 / 2$ and positive if $1 / 2<H<1$. The sample paths of FBM series are relative more rough and variable for small Hurst index $H$, while the sample paths of FBM series are more smooth for large Hurst index $H$.

In this work, we try to reveal the relationship between FBM and its related recurrence network from the perspective of network structure. This prompted us to further study the fundamental topological and fractal properties of recurrence networks constructed from FBMs. We think that applying recurrence network analysis (RNA) to FBM can yield results which are theoretically meaningful. The reasons are: (a) Visibility graphs and recurrence networks are two main classes of methods to map time series to networks. Recently some works have been done to study the relationship between the exponents of newly developed methods(including visibility graphs) and the Hurst index of the associated FBMs [24-26]. (b) Donges et al. [21] have proposed an analytical framework for RNA of time series of chaotic maps and stochastic processes (such as uniformly distributed noise and Gaussian noise). FBMs are generalization of Gaussian noise. (c) Donner et al. [22] applied RNA to study the real economic time series. And it is well known that FBM has been used as a theoretical framework to study economic time series [23]. In 1999, Riley et al. [27] applied the RQA technique for analyzing center of pressure (COP) signals and the nonstationarity of the COP is expected from a consideration of COP trajectories as FBM.

The remaining of this paper is organized as follows. In Section 2, we adopt the recurrence plot method to construct recurrence networks. In Section 3, we investigate the basic topological characteristics of the recurrence networks constructed from FBMs. We next introduce the random sequential box-covering method [28] to calculate the fractal dimension of the recurrence networks in Section 4. In Section 5, we introduce an improved algorithm newly developed by our group [29], which is based on the modified fixed-size box-counting algorithm [30], to probe the multifractal behavior of recurrence networks. We finally draw some conclusions in Section 6 .

\section{Recurrence network}

The method of recurrence plots is based on the theory of phase space reconstruction introduced by Packard et al. [31]. In order to easily construct a more suitable space and then to reveal more meaningful information from original time series, Packard et al. [31] proposed the derivative reconstruction method and time delay method to reconstruct a finite-dimensional phase space. However, in practice, we do not know any prior information of time series, therefore the latter method is widely used to reconstruct phase space of time series. For a given scalar time series $\left\{x_{i}, i=1,2, \cdots, L\right\}$, we construct the delay vectors

$$
X(i)=\left(x_{i}, x_{i+\tau}, \cdots, x_{i+(m-1) \tau}\right), X(i) \in \mathbf{R}^{m},
$$

for $i=1,2, \cdots, N$, where $N=L-(m-1) \tau, m$ is the embedding dimension and $\tau$ is the time delay. Takens' embedding theorem ensures that we can recreate a topologically equivalent $m$-dimensional phase space from an infinite noise-free time series data by means of the time delay method [32]. 
The most significant advantage of phase space reconstruction is that it can preserve the geometrical invariants of the original system, such as the fractal dimension and the Lyapunov exponents [32]. So we can investigate much about the dynamics characteristics of time series in phase space more easily. The basic embedding theorem of phase space reconstruction assumes that we can choose the delay time $\tau$ without any limitation and the embedding dimension $m$ under the condition of $m \geq 2 d+1$, where $d$ is the fractal dimension of the underlying attractor. However, since the observed time series data sets are finite and noisy in the most common case, the selection of the time delay $\tau$ and the embedding dimension $m$ is rather important for the quality of the phase space reconstruction.

The binary recurrence matrix $R_{N N}=\left(R_{i, j}\right)_{N N}$ is defined as

$$
R_{i, j}=\Theta(\varepsilon-\|X(i)-X(j)\|), i, j=1,2, \cdots, N
$$

where $\varepsilon$ is a threshold distance, $\|\cdot\|$ is a suitable norm (e.g., the $L_{1}$ - norm, the Euclidean norm and the $L_{\infty}$ - norm) in the considered phase space and $\Theta(x)$ is the Heaviside step function (i.e. $\Theta(x)=0$ if $x<0$, and $\Theta(x)=1$ otherwise). The resulting matrix $R_{N N}$ is a symmetric matrix where the elements $R_{i, j}=0$ or 1 . Moreover, the matrix $R_{N N}$ exhibits the line of identity (the main diagonal) $R_{i, i}=1$. In a two-dimensional space, the recurrence plot is described by using different colors for different values of the recurrence matrix, e.g., plotting a black dot at the coordinates $(i, j)$ if $R_{i, j}=1$, and a white dot if $R_{i, j}=0$. Thus the recurrence plot always has a black main diagonal line.

Now we obtain the adjacency matrix $A_{N N}=\left(A_{i, j}\right)_{N N}$ of the recurrence network from the recurrence matrix $R_{N N}$ as

$$
A_{i, j}=R_{i, j}-\delta_{i, j},
$$

where $\delta_{i, j}$ is the Kronecker delta function introduced here in order to avoid self-loops [3]. In complex network, each state vector $X(i)$ of the reconstructed phase space represents a single node. The topological structure of the recurrence network can be described with the adjacency matrix $A_{N N}$, and the elements $A_{i, j}=1$ and $A_{i, j}=0$ correspond to connection and disconnection, respectively. And the size of the network is $N$.

As mentioned above, the choice of the time delay $\tau$ and the embedding dimension $m$ plays an important role in the recurrence plot method. If the time delay $\tau$ selected is too small, each coordinate of the vector $X(i)$ will be so close to each other that the trajectories of the reconstructed phase space are compressed along the identity line, and this phenomenon is referred to as redundance. If the time delay $\tau$ selected is too large, the coordinates of the vector $X(i)$ are completely independent of each other and then the reconstructed attractor dynamics become causally disconnected, and this phenomenon is referred to as irrelevance [33]. At present, auto-correlation function [34] and mutual information method [35] are the most common approaches for the estimation of the time delay $\tau$. Fraser et al. [35] proposed a recursive method of calculating mutual information and pointed out that the first local minimum of mutual information is the best criterion for choosing time delay $\tau$ in phase space reconstruction from time series data.

Takens' embedding theorem gives us a way to estimate the embedding dimension $m$. This is to say that all self-intersections of the orbit (which is the reconstructed attractor) in the reconstructed 
phase space will be eliminated for any embedding dimension $m \geq 2 d+1$. The attractor will be completely unfolded in this embedding dimension $m$. But in practice, since the dimension $d$ is unknown in many cases, we cannot directly calculate the embedding dimension $m$ from the Takens' embedding theorem. Many algorithms based on computing some invariants on the attractor have been proposed to calculate the minimum embedding dimension $m$. The most popular method for calculating the minimum embedding dimension is the False nearest-neighbors (FNN) algorithm [36, 37]. According to the Takens' embedding theorem, we assume that $m$ is qualified as an embedding dimension. If any two points are close to each other in the $m$-dimensional phase space, then they are still close to each other in the $(m+1)$-dimensional phase space. We call this two points true neighbors, otherwise, they are false neighboring points. The main idea of the FNN algorithm is to measure the percentage of false nearest neighbors along a signal trajectory change with increasing embedding dimension. The optimal embedding dimension means that there is no false neighbors and self-intersections in the phase space. This indicates that the minimum embedding dimension is that for which the percentage of false nearest neighbors drops to zero or nearly zero for a given tolerance level for the first time [36].

The threshold distance $\varepsilon$ is another key parameter of an RP. The selection of the threshold $\varepsilon$ depends strongly on the system considered. If $\varepsilon$ is too small, there may be almost no recurrence points and we cannot learn anything about the recurrence structure of the underlying system. On the other hand, if $\varepsilon$ is too large, almost every point is a neighbor of every other point, which leads to a lot of artifacts and redundant information [38]. Therefore, special attention has been required on its choice. In practice, the most common method is to set $\varepsilon$ for a certain proportion of the standard deviation $\sigma$ of the original time series. However, Riley and Van Orden suggested choosing $\varepsilon$ such that the recurrence point density remains low (often smaller than 5\%) [39]. This method is often used in RQA. The advantage of choosing an adaptive recurrence threshold by using a fixed recurrence rate in RQA is that it can preserve the recurrence point density and then allows us to compare the results of recurrence quantification analysis of different systems. It is easy to imagine that there is no direct relationship between recurrence rate or link density and network connectivity. And it is so difficult for us to find a fixed recurrence rate that all recurrence networks obtained at recurrence threshold $\varepsilon$ calculated from the fixed recurrence rate are connected. However, we restrict our attention to the consideration of connected network in this article. Therefor we do not adopt the fixed recurrence rate for obtaining such an appropriate recurrence threshold. If we do so, then we will not be able to study the topological and fractal properties of the entire network. In other word, we can only focus on the disconnected components or the largest connected component of each recurrence network.

The critical phenomenon is one of the most interesting findings of complex networks. In 1959, Erdös et al. [40] introduced the classical random graph model and studied the structural phase transition of the birth of the giant connected component in the network architectures. Every pair of nodes of random graph is connected with probability $p$. They found that there exists a critical probability $p_{c}$ where a giant connected component was formed and it can be spread across the entire network. If the probability $p$ is less than this critical probability $p_{c}$, the network is composed 
of disconnected components. Similarly to the critical behavior of random-graph theory, we study the critical connectivity of the recurrence networks by examining the size of the largest connected component and then determine the critical recurrence threshold $\varepsilon_{c}$. Here we firstly employ the connection rate of network to quantify the connectivity of the recurrence network, which is defined as the number of nodes of the largest connected component divided by the size of the network. Secondly, we observe the change of connection rate of network with the increase of the parameter $\varepsilon$. It can be easily imagined that the connection rate of network increases when the parameter $\varepsilon$ becomes larger, as illustrated in Fig. 1. In Fig. 1, we set the recurrence threshold $\varepsilon$ ranging from $0.01 \sigma$ to $0.3 \sigma$ with a step of $\sigma_{s}=0.01 \sigma$, where $\sigma$ is the standard deviation of the original FBM series. Finally, we choose an appropriate recurrence threshold $\varepsilon_{c}$ where the connection rate of the network arrives at 1 for the first time. As can be seen in Fig. 1, we set the critical recurrence threshold $\varepsilon_{c}=0.12 \sigma$. The recurrence network obtained at recurrence threshold $\varepsilon_{c}$ will be connected. The threshold $\varepsilon$ beyond this critical value $\varepsilon_{c}$ will result in redundant connections among nodes, and threshold $\varepsilon$ below this critical value $\varepsilon_{c}$ will lead to a number of disconnected components where each component only contains a connected subnetwork. Consequently, we select this suitable recurrence threshold $\varepsilon_{c}$ to construct the recurrence network and then investigate its topological and fractal properties. In addition, we also notice that the critical recurrence threshold $\varepsilon_{c}$ decreases with the increase of the Hurst index $H$ from 0.4 to 0.95 . We know that the FBM series is relatively more smooth and regular for large Hurst index $H$. The consequence is that there are many data points in the original FBM series are mapped to state vectors which are close to each other in a dense region of the phase space so that the largest connected component of the recurrence network contains more nodes than the one of the network with the small Hurst index $H$. The specific explanation for this phenomenon can be found in the second subsection of the Section 3.

In this work, we consider FBM of length $L=2^{12}$ with different Hurst indices $H$ ranging from 0.05 to 0.95 (the step difference is 0.05 ). Recurrence networks can be constructed by the following steps:

(i) For a given FBM time series, we use the mutual information method [35,41] and the FNN algorithm [36] to calculate the time delay $\tau$ and the embedding dimension $m$, respectively. These two procedures are available on TOCSY software platform [17]. The values of embedding dimensions $m$ we got vary from 5 to 7 , and those of the time delay $\tau$ vary from 10 to 20.

(ii) We use the connection rate of the recurrence network to determine the suitable threshold distance $\varepsilon_{c}$ [42]. We observe the connection rate of network reaching 1 for the first time when the parameter $\varepsilon$ ranging from $0.01 \sigma$ to $0.8 \sigma$ with a step of $\sigma_{s}=0.01 \sigma$, where $\sigma$ is the standard deviation of the original FBM series. The connection rate of network can be calculated by Matlab-BGL toolbox [43] in Matlab.

(iii) Based on the above steps, we can calculate the recurrence matrix $R_{N N}$ by Eq. (2). Then we obtain the binary adjacency matrix $A_{N N}$ of an unweighted and undirected recurrence network by Eq. (3). As a result, the recurrence network is also connected. 
For clarity of visualization, we here only draw the recurrence network for the FBM series of length $L=2^{8}$ with Hurst index $H=0.6$. After obtaining the adjacency matrix $A_{N N}$ of recurrence network according to the above three steps, we convert the adjacency matrix $A_{N N}$ into "Pajek" [44] format and then we adopt the "Pajek" which is a freely available software platform for visualizing and analyzing complex networks to visualize the recurrence network. As can be clearly seen in Fig. 2 , the recurrence network constructed from the associated FBM series is connected.

In addition, we find that the connection rates of recurrence networks constructed from FBMs with Hurst indices $H=0.05,0.1, \cdots, 0.3$, and 0.35 are far less than 1 . In these cases, the recurrence networks are not connected. So in the following sections, we only consider connected networks with Hurst indices $H$ from 0.4 to 0.95 .

\section{Fundamental topological properties}

In this section, we numerically study the basic topological features of recurrence networks including the degree distribution, the clustering coefficient and the motif distribution. Recent works have shown that a large number of real-world networks are referred to as scale-free networks because the degree distributions $P(k)$ follows a power-law

$$
P(k) \sim k^{-\alpha},
$$

where the degree exponent $\alpha$ varying in the range $2<\alpha<3[5,6,45]$. The degree distributions of the visibility graphs constructed from FBMs have power-law tails [8,24,46]. In addition, Xie et al. [25] found that horizontal visibility graphs constructed from FBMs have exponential degree distributions

$$
P(k) \sim e^{-\lambda k},
$$

and the degree exponent $\lambda$ increases with Hurst index $H$.

It has been shown that many real-world complex networks share similar universal statistical features, such as the small-world character and the scale-free distribution, but may demonstrate different local structure characteristics $[4,5,47]$. An in-depth study of motif distribution can help us to understand the design principles of complex networks on the local structure level $[47,48]$. In 2004, Milo et al. [48] developed an approach for comparing network local structures, basing on the significance profile (SP). They calculated the triad significance profile (TSP) for networks from different fields and found that networks with similar characteristic profiles are grouped into superfamilies.

\subsection{Degree distribution}

Degree distribution is one of the most fundamental and important topological properties of complex networks. We simulated FBMs with different Hurst indices $H$ ranging from 0.40 to 0.95 with an increment of 0.05 . For each value of $H$, we generated 1000 realizations and then calculated the average degree distribution. For example, we give the average degree distribution of recurrence networks constructed from FBMs with Hurst index $H=0.6$ in Fig. 3. We find that all the 
recurrence networks constructed from FBM series exhibit exponential degree distributions. We estimated the degree exponents $\lambda$ and display the average degree exponents $\langle\lambda\rangle$ in Fig. 4. From Fig. 4, we can see that the parameter $\lambda$ increases first and then decreases with the increase of $H$. The inflection point is at around $H=0.8$. We find that the relationship between $H$ and $\langle\lambda\rangle$ can be fitted by a cubic polynomial function which is also shown in Fig. 4.

\subsection{Clustering coefficient}

The clustering coefficient measures the density of triangles in a complex network. The definition of the clustering coefficient given by Watts and Strogatz in Ref. [4] has been used quite widely in complex network. The local clustering coefficient $C_{i}$ is defined as

$$
C_{i}=\frac{2 E_{i}}{k_{i}\left(k_{i}-1\right)}=\frac{\sum_{j, h} A_{i, j} A_{j, h} A_{h, i}}{k_{i}\left(k_{i}-1\right)},
$$

where $k_{i}$ is the degree of node $i, E_{i}$ is the actual number of edges among $k_{i}$ nearest neighbor nodes of the node $i$. The clustering coefficient of the whole network is then given by the average of $C_{i}$ over all the nodes in the entire network:

$$
C=\frac{1}{N} \sum_{i} C_{i}
$$

From the geometrical point of view, the local clustering coefficient $C_{i}$ also can be defined as

$$
C_{i}=\frac{\text { number of triangles connected to vertex } i}{\text { number of triples centered on vertex } i}
$$

where a triple represents a vertex with edges connected any two neighbor vertices. There are two different triples as shown in Fig. 5. The triple $T_{1}$ will occur if the three vertices are all close to each other. Conversely, the triple $T_{2}$ will occur if the vertex $i$ is connected to its two neighbor vertices $j$ and $k$, but vertices $j$ and $k$ are not connected. In network topology, the triangle structure $T_{1}$ means transitivity and stability in the network.

In this paper, we study the dependence of the clustering coefficient $C$ of the recurrence networks against the Hurst index $H$ of the original FBMs. For each $H$, we simulated the FBM 1000 times, hence we got 1000 FBM series. For each FBM series, a recurrence network was constructed and its clustering coefficient $C$ was calculated. We display the relationship between $H$ and the average clustering coefficient $\langle C\rangle$ of recurrence networks in Fig. 6. From Fig. 6, we can see that the average clustering coefficient $\langle C\rangle$ increases with the Hurst index $H$. The results show that there are more triangle structures in the recurrence network when the Hurst index $H$ increases from 0.4 to 0.95. This means that the transitive and stable structure is more common for large Hurst indices. This phenomenon can be easily explained by following points. It is well known that the Hurst index characterizes the raggedness and irregularity of FBM. As already mentioned, the FBM is a self-similar process with stationary increments and possesses long-range dependence. The Hurst index $H(0<H<1)$ is a measure of the intensity of long-range dependence in a FBM series. More

specifically, the FBM with $H=0.5$ indicates the absence of long-range dependence. And the closer Hurst index $H$ is to 1 , the greater the extent of long-range dependence or persistence. The Hurst 
index $H$ less than 0.5 corresponds to anti-persistence, which means that the process displays strong negatively correlated and fluctuates violently. For large Hurst index $H$, the FBM series is relatively more smooth and regular. The result is that close data points in the original FBM series are mapped to state vectors $X(i)$ which are close to each other in a dense region of the phase space so that these nodes $X(i)$ are more likely to be connected to each other in recurrence network. As expected, there are many more transitive and stable structures $T_{1}$ are to appear in recurrence network constructed from the FBM series with large Hurst index $H$. However, the FBM series with small Hurst index $H$ is relatively more rough and irregular. As a consequence of this, these close points in the original FBM series are mapped to state vectors $X(i)$ in a sparse region of the phase space that are less likely to be mutually connected, and therefore the triple $T_{2}$ will be more common. From this point of view, the recurrence network reflects the smoothness and regularity of the FBMs to some extent.

\subsection{Motif distribution}

Because it is very time-consuming to study next a few properties of large complex networks, we only generated 100 realizations of FBM for each value of Hurst index $H$ and calculated the average in the following.

Researches have shown that subgraphs or motifs are the building blocks of complex networks and the superfamilies of networks can be defined as the network motif patterns of occurrence $[47,48]$. Distinct from the degree distribution and the clustering coefficient, the occurrence frequency of small subgraphs or motifs can characterize the local structural properties of real-world networks and then map the relation between the function and the local structure of real-world systems [47]. $\mathrm{Xu}$ et al. [12] observed the superfamily phenomenon in the nearest-neighbor networks constructed from different time series. They also found that the distribution of network motif ranks can be used to distinguish and to characterize different types of dynamics in periodic, chaotic and periodic with noise processes [12]. Xie et al. [25] calculated the frequencies of occurrence of the six motifs of size 4 within the horizontal visibility graphs constructed from FBMs.

In this work, the constructed recurrence networks are connected and undirected, so here we only consider motifs with 4 nodes as in Ref. [25]. Fig. 7 shows all six different network motifs of size 4 in connected and undirected networks. These motifs reflects the different local structure characteristics in network. There are two extreme blocks: motif $M_{1}$ and motif $M_{6}$. The motif $M_{1}$ means the most irregular and nontransitive structure that will appear if the node is connected to its three neighbor nodes, but these neighbors are not connected to each other. On the contrary, the motif $M_{6}$ indicates the most transitive structure that will appear if the three neighbors are also connected to each other. The relative occurrence frequencies of motifs [25] are defined as

$$
P(M)=\frac{n(M)}{\sum_{M=M_{1}, \ldots, M_{6}} n(M)},
$$

where $n(M)$ is the number of motif $M$ in the network. After constructing the recurrence networks based on the recurrence plot method described above, we calculated the occurrence frequencies of various motifs within the recurrence networks by the network motif detection tool provided by Milo et al. [49]. For each Hurst index $H$, which runs from 0.40 to 0.95 with a step of $0.05,100$ realizations 
of FBMs were generated. Then we constructed these time series into 100 recurrence networks and obtained the average of $P(M)$.

In Fig. 8, we rearrange the relative occurrence frequencies $\langle P(M)\rangle$ in descending order for different Hurst indices $H$. As we can see from Fig. 8, these seemingly unrelated recurrence networks are divided into two superfamilies based on the very similar motif rank distributions. Fig. 8(a) shows a superfamily determined by the motif rank $M_{2} M_{3} M_{1} M_{5} M_{6} M_{4}$, which includes the recurrence networks constructed from FBMs with Hurst indices $H=0.4,0.45,0.5,0.55$, and 0.6. The rest of recurrence networks with Hurst indices $H=0.65, \cdots, 0.95$ belong to another superfamily and the motif rank pattern is $M_{2} M_{3} M_{5} M_{6} M_{1} M_{4}$ (Fig. 8(b)). In addition, three particular motifs $M_{1}, M_{5}$, and $M_{6}$ play an important role in the classification of recurrence networks we constructed because their order determines the motif rank distributions. A similar phenomenon was also observed in Refs. $[12,13]$. They gave a detailed explanation for this common phenomenon. Fig. 9 further illustrates the dependence of $\langle P(M)\rangle$ with respect to the Hurst index $H$. More specially, the occurrence frequency $\left\langle P\left(M_{1}\right)>\right.$ of motif $M_{1}$ strictly monotonically decreases with the increase of the Hurst index $H$ (see Fig. 9(a)). In contrast to the motif $M_{1}$, the frequencies $\left\langle P\left(M_{5}\right)\right\rangle$ and $<P\left(M_{6}\right)>$ increase with the increase of the Hurst index $H$ (see Fig. 9(e) and Fig. 9(f)). We also note that the fluctuation of the frequencies $\left\langle P\left(M_{4}\right)\right\rangle$ is comparatively small in Fig. $9(\mathrm{~d})$, which means that the motif $M_{4}$ almost does not appear in all of the recurrence networks. These results indicate that there are more regular and transitive structures in the networks when the Hurst index $H$ varies from 0.4 to 0.95 . That is to say, the fully transitive motif $M_{6}$ will be common in these recurrence networks constructed from the FBM series with large Hurst index $H$ than the ones with small Hurst index $H$. On the contrary, the irregular and nontransitive structure such as $M_{1}$ will be more common for these network with small Hurst index $H$. These trends are roughly coincide with the ones in the previous subsection. It should be noted that two triples $T_{1}$ and $T_{2}$ in Fig. 5 are just two motifs with 3 nodes in undirected network. Measuring and analyzing the density distribution of the motifs with 4 nodes may be seen as a generalization of the aforementioned transitivity concept. Therefore, as mentioned in the previous subsection, we can give a similar explanation for

these trends in this subsection. In this sense, recurrence networks capture the correlation of the associated FBMs.

\section{Fractal dimension}

In 1967, Mandelbrot introduced the fractal idea in Ref. [50]. In fractal geometry, a fractal object is self-similar because it contains small parts similar to the whole [51,52]. Recently, the fractal and self-similarity properties of complex networks have been studied extensively in various fields and systems $[30,53,54]$. Song et al. [55] found that many real-world networks such as the world-wide web (WWW), social networks, protein-protein interaction (PPI) networks and cellular networks consist of self-repeating patterns. They also believed that these complex networks are self-similar under a certain length-scale. After the small-world character and scale-free property, self-similarity has become the third basic characteristic of complex networks. 
To gain further understanding of complex networks, numerous algorithms have been developed to calculate the fractal dimension of complex networks. Song et al. [56] proposed to calculate the fractal dimension via a box-counting method. Kim et al. [28, 54] investigated the skeleton and fractal scaling in complex networks using an improvement algorithm which is a modified version of the original method introduced by Song et al. Then Zhou et al. [57] developed an alternative algorithm, based on the edge-covering box-counting, to detect self-similarity of cellular networks. In this section, we adopt the random sequential box-covering method proposed by Kim et al. [28] for calculating the fractal dimensions of recurrence networks.

For a given network, let $N_{B}\left(l_{B}\right)$ be the smallest number of boxes of lateral size $l_{B}$ which are needed to cover the entire network. The fractal scaling implies the power-law relationship between $N_{B}\left(l_{B}\right)$ and $l_{B}$; the fractal dimension $d_{B}$ is then given by

$$
N_{B}\left(l_{B}\right) \sim l_{B}^{-d_{B}}
$$

Usually, the fractal dimension $d_{B}$ can be obtained by fitting the linear relationship between $N_{B}\left(l_{B}\right)$ and $l_{B}$ in a log-log plot.

Before introducing the random sequential box-covering algorithm [28], we use Floyd's algorithm [58] of Matlab-BGL toolbox [43] to calculate the shortest-path distance matrix $D$ for each network according to the adjacency matrix $A_{i, j}$ of the recurrence network. The random sequential boxcovering algorithm [28] can be described as follows. We start with all vertices labelled as not burned. Then,

(i) Select a vertex randomly at each step; this vertex serves as a seed and then consider the seed as the center of a box.

(ii) For the center of the box, search all the neighbor vertices within distance $l_{B}$ and burn all vertices which are found but have not been burned yet. Assign the newly burned vertices to the new box. If no newly burned vertex is found, then this box is discarded.

(iii) Repeat Steps (i) and (ii) until all vertices in the entire network are assigned to their respective boxes.

In this work, we simulated FBM series with different Hurst indices $H$ ranging from 0.4 to 0.95 in the step of 0.05 . For each $H$, we simulated 100 FBM time series and then constructed the obtained series into 100 recurrence networks. Apparent power-law behaviors of the two typical empirical recurrence networks constructed from FBM series with different Hurst indices are shown in Fig. 10. The fractal dimension $d_{B}$ is the absolute value of the slope of linear regression between $\ln N_{B}\left(l_{B}\right)$ and $\ln l_{B}$ for each Hurst index. Fig. 11 shows the relationship between Hurst index $H$ and the average fractal dimension $\left\langle d_{B}>\right.$ over 100 realizations. As we can see from Fig. 11, the average fractal dimension $\left\langle d_{B}>\right.$ decreases with increasing $H$. Furthermore, it is surprising that the curve shows a nice linear relationship:

$$
<d_{B}>=2.0064-1.0441 H
$$


which approximates the theoretical relationship between Hurst index $H$ and the fractal dimension $d$ of the graph of FBM $d=2-H$. Our numerical results show that the fractal dimension of the recurrence networks constructed is very close to that of the graph of the original FBMs. In other words, the fractality of FBMs is inherited in their recurrence networks. We can explain here why the two fractal dimensions are so closely related that the Hurst index $H$ of the FBM series can be approximately estimated from the perspective of the complex network. As it has already been mentioned, the pairs of nodes in the recurrence network can be connected only when the two corresponding state vectors $X(i)$ in phase space are close enough so that the phase space distance between them is less than the critical recurrence threshold $\varepsilon_{c}$. This is to say that recurrence networks we constructed are random geometric graphs faithfully representing the geometry of a set in phase space. Hence, neighborhood sizes around a node in the network as well as around the corresponding state vector in phase space will show the same scaling behavior. This results in analogous behavior of the derived fractal dimensions.

So far, we have numerically studied the basic topological properties and the fractal dimensions of the recurrence network. More recently, some chaotic model systems such as logistic map, Hénon map, generalised baker's map and Bernoulli map, periodic and two-dimensional quasiperiodic motions have been studied analytically by Donner et al. [59] and Donges et al. [21] from the point of view of $\varepsilon$-recurrence network. To better understand the interrelationships between network properties and dynamical system, they defined some newly continuous measures based on well-known graph theoretical measures and gave two novel notions of dimension in phase space (clustering dimension and transitivity dimension). They have put forward a theoretical framework for these new measures. Their results indicated that $\varepsilon$-recurrence networks show a strong relation between dynamical systems and graph theory $[21,59]$. Their work provides us with a possible way to derive the analytical results of these topological properties and dimensions for recurrence network of FBM series in our future work.

\section{Multifractal analysis}

In real world, two fractal objects may have the same fractal dimension but looks completely different. For these real-world fractals, however, the tool of multifractal analysis shows better performance and seems more powerful than fractal analysis. Multifractal analysis has been successfully applied in a variety of fields such as financial modelling [60,61], biological systems (e.g. [62-65]) and geophysical data analysis (e.g. [66,67]). Wang et al. [30] applied the modified fixed-size box-counting algorithm to explore the multifractal behavior of some theoretical networks, namely scale-free networks, smallworld networks, random networks, and a kind of real networks, namely PPI networks of different species. Their numerical results indicate that multifractality exists in scale-free networks and PPI networks, while for small-world networks and random networks their multifractality is not clear-cut.

Fixed-size box-covering algorithm [68] is one of the most common and important methods of multifractal analysis. For a given measure $\mu$ with support set $E$ in a metric space, we consider the 
partition sum

$$
Z_{\epsilon}(q)=\sum_{\mu(B) \neq 0}[\mu(B)]^{q},
$$

where $q \in R$ and the sum runs over all different nonempty boxes $B$ of a given size $\epsilon$ in a covering of the support set $E$. From the definition above, we can obtain $Z_{\epsilon}(q) \geq 0$ and $Z_{\epsilon}(0)=1$. The exponent $\tau_{1}(q)$ of the measure $\mu$ can be defined as

$$
\tau_{1}(q)=\lim _{\epsilon \rightarrow 0} \frac{\ln Z_{\epsilon}(q)}{\ln \epsilon}
$$

and the generalized fractal dimensions of the measure $\mu$ are defined as

$$
D(q)=\frac{\tau_{1}(q)}{q-1}
$$

for $q \neq 1$, and

$$
D(q)=\lim _{\epsilon \rightarrow 0} \frac{Z_{1, \epsilon}}{\ln \epsilon},
$$

for $q=1$, where $Z_{1, \epsilon}=\sum_{\mu(B) \neq 0} \mu(B) \ln \mu(B)$. The linear regression of $\left[\ln Z_{\epsilon}(q)\right] /(q-1)$ against $\ln \epsilon$ for $q \neq 1$ gives numerical estimates of the generalized fractal dimensions $D(q)$, and similarly a linear regression of $Z_{1, \epsilon}$ against $\ln \epsilon$ for $q=1$. In particular, $D(0)$ is the box-counting dimension (or fractal dimension), $D(1)$ is the information dimension, and $D(2)$ is the correlation dimension. If the $\tau_{1}(q)$ or $D(q)$ curve versus $q$ is a straight line, the object is monofractal. However, if this curve is convex, the object is multifractal.

In order to calculate the exponent $\tau_{1}(q)$ and the generalized fractal dimensions $D(q)$ and then study the multifractality of networks, the measure $\mu$ of each box is usually defined as the ratio of the number of nodes covered by the box and the total number of nodes in the entire network. Wang et al. [30] proposed a modified fixed-size box-counting algorithm to calculate the $\tau_{1}(q)$ and $D(q)$ and then investigate the multifractal behavior of complex networks. Recently Li et al. [29] made some improvements based on the modified fixed-size box-counting algorithm proposed by Wang et al. [30]. The improved algorithm [29] can be summarized as follows:

(i) Initially, make sure all nodes in the entire network are not covered and no node is selected as a center of a box.

(ii) According to the size $N$ of networks constructed, set $t=1,2, \cdots, 1000$ appropriately. Rearrange the nodes into 1000 different random orders. More specifically, in each random order, nodes which will be selected as a center of a box are randomly arrayed.

(iii) Set the radius $r$ of the box which will be used to cover the nodes in the range $r \in[1$, $d]$, where $d$ is the diameter of the network.

(iv) Treat the nodes in the $t$-th random order that we got in (ii) as the center of a box, search all the neighbor nodes by distance $r$ from the center and cover all nodes which are found but have not been covered yet.

(v) If no newly covered nodes have been found, then this box is discard. 
(vi) Repeat Steps (iv) and (v) until all the nodes are covered by the corresponding boxes. We denote the number of boxes in this box covering as $N(t, r)$.

(vii) Repeat Steps (iv) to (vi) for all 1000 random orders to find a box covering with minimal number of boxes $N(t, r)$.

(viii) For the nonempty boxes $B$ in the first box covering with minimal number of boxes $N(t, r)$, define the measure of this box as $\mu(B)=N_{B} / N$, where $N_{B}$ is the number of nodes covered by the box $B$, and $N$ is the size of the network. then calculate the partition $\operatorname{sum} Z_{r}(q)=$ $\sum_{\mu(B) \neq 0}[\mu(B)]^{q}$.

(ix) For different value of $r$, repeat Steps (iii) to (viii) to calculate the partition sum $Z_{r}(q)$ and then use the $Z_{r}(q)$ for linear regression.

A key step of linear regression is to obtain the appropriate range of $r \in\left[r_{\min }, r_{\max }\right]$. Then we calculate the exponents $\tau_{1}(q)$ and generalized fractal dimensions $D(q)$ in the scaling ranges. In our calculation, we obtain the generalized fractal dimensions through a linear regression of $\left[\ln Z_{r}(q)\right] /(q-1)$ against $\ln (r / d)$ for $q \neq 1$, and similarly a linear regression of $Z_{1, r}$ against $\ln (r / d)$ for $q=1$, where $Z_{1, r}=\sum_{\mu(B) \neq 0} \mu(B) \ln \mu(B)$.

Fig. 12 shows the linear regression for the recurrence network constructed from FBM with Hurst index $H=0.6$. The numerical results show that the best fit occurs in the range $r \in(2,10)$ for this case $(H=0.6)$. We select the best linear fit scaling range to calculate the exponents $\tau_{1}(q)$ and generalized fractal dimensions $D(q)$ and then to determine the multifractality of recurrence networks from the shape of these curves.

In this paper, we detect the multifractal behavior of recurrence networks using our improved version of the modified fixed-size box-counting algorithm introduced by Li et al. [29]. For each value of the Hurst index $H$, we averaged the results over 100 realizations of the FBM series. We summarize the corresponding numerical results in Table 1 , which includes the Hurst index $H$, the average box-counting dimension $\langle D(0)\rangle$, the average information dimension $\langle D(1)\rangle$, the average correlation dimension $\langle D(2)>$, and $\Delta D(q)$, where the quantity

$$
\Delta D(q)=\max D(q)-\min D(q)
$$

was used to verify how $D(q)$ changes along each curve. From Table 1, we can see that the average box-counting dimension $\langle D(0)\rangle$, the average information dimension $\langle D(1)\rangle$ and the average correlation dimension $\langle D(2)>$ are roughly decrease with the increase of the Hurst index $H$ from 0.4 to 0.95 . We show the average $\left\langle\tau_{1}(q)\right\rangle$ curves in Fig. 13 and average $\langle D(q)\rangle$ curves in Fig. 14 . From Figs. 13 and 14, we find that the $\left\langle\tau_{1}(q)\right\rangle$ and $\langle D(q)\rangle$ curves of recurrence networks are not straight lines. So the multifractality exists in these recurrence networks constructed from FBM series. Meanwhile, we also find that the multifractality of these networks becomes stronger at first and then weaker which indicated by the value of $\Delta D(q)=D(-10)-D(10)$ when the Hurst index of the associated time series becomes larger from 0.4 to 0.95 . In particular, the recurrence network with the Hurst index $H=0.5$ possess the strongest multifractality. The dependence relationships 
of the average information dimension $\langle D(1)\rangle$ and the average correlation dimension $<D(2)>$ on the Hurst index $H$ are given in Fig. 15. As shown in Fig. 15, we find that these relationship can be well fitted by following linear formulas:

$$
<D(1)>=1.7825-0.9046 H
$$

and

$$
<D(2)>=1.5736-0.7956 H \text {. }
$$

\section{Conclusions}

In this work, we constructed recurrence networks from FBM series based on the idea of recurrence plot. We extracted the statistical properties of time series from the perspective of complex networks. We studied the basic topological features of the recurrence networks constructed from FBM series with different Hurst indices $H$. Our numerical results indicate that the recurrence networks constructed exhibit exponential degree distributions. With the increase of $H$, the average degree exponent $\langle\lambda\rangle$ increases first and then decreases. The relationship between $H$ and $\langle\lambda\rangle$ can be fitted by a cubic polynomial function. It was found that the average clustering coefficient $<C>$ increases with the Hurst index $H$, which means that there are more transitive and stable structures for networks constructed from FBMs with large Hurst index $H$. At the microscopic level, we investigated the motif rank distribution of recurrence networks. We found that the recurrence networks are grouped into two superfamilies based on the motif rank distribution. The three key motifs $M_{1}, M_{5}$, and $M_{6}$ determine the motif rank pattern and then classify networks. At the same time, we also paid attention to the dependence relationship of the average occurrence frequency $<P(M)>$ with respect to the Hurst index $H$.

From the aspect of fractality and self-similarity, we performed fractal and multifractal analyses using the random sequential box-covering method and the improved method based on modified fixed-size box-counting algorithm, respectively. The numerical results show that the average fractal dimension $\left\langle d_{B}>\right.$ decreases with Hurst index $H$ and the linear relationship $\left\langle d_{B}>\approx 2-H\right.$ was obtained surprisingly. Moreover, our numerical results of multifractal analysis show that the multifractality exists in these recurrence networks, and the multifractality of these networks becomes stronger at first and then weaker when the Hurst index of the associated time series becomes larger from 0.4 to 0.95 . In particular, the recurrence network with the Hurst index $H=0.5$ possess the strongest multifractality. We also noted that the average information dimension $\langle D(1)\rangle$ and the average correlation dimension $\langle D(2)>$ are roughly decrease with the increase of the Hurst index $H$ from 0.4 to 0.95 . The dependence relationships of the average information dimension $\langle D(1)\rangle$ and the average correlation dimension $\langle D(2)\rangle$ on the Hurst index $H$ can be well fitted with linear functions. From the above results, we conclude that the inherent nature of time series affects the structure characteristics of the associated networks and the dependence relationship between them appears retained. Our works support that complex networks is a suitable and effective tool to perform time series analysis. 


\section{Acknowledgments}

This project was supported by the Natural Science Foundation of China (Grant nos. 11071282 and 11371016), the Chinese Program for Changjiang Scholars and Innovative Research Team in University (PCSIRT) (Grant No. IRT1179), the Research Foundation of Education Commission of Hunan Province of China (grant no. 11A122), the Lotus Scholars Program of Hunan province of China, the Aid program for Science and Technology Innovative Research Team in Higher Educational Institutions of Hunan Province of China.

\section{References}

[1] R. Albert and A.L. Barabási, Rev. Mod. Phys., 74 (2002) 47-97.

[2] R.V. Donner, Y. Zou, J.F. Donges, N. Marwan, and J. Kurths, Phys. Rev. E, 81 (2010) 015101(R).

[3] R.V. Donner, Y. Zou, J.F. Donges, N. Marwan, and J. Kurths, New J. Phys., 12 (2010) 033025.

[4] D.J. Watts and S.H. Strogatz, Nature, 393(6684) (1998) 440-442.

[5] A.L. Barabási and R. Albert, Science, 286(5439) (1999) 509-512.

[6] M.E.J. Newman, SIAM Rev, 45 (2003) 167-256.

[7] M. Small, J. Zhang, and X.K. Xu, in: Lect. Notes Institue Comput. Sci. Soc. Infor. Telecommun. Engineering, 5 (2009) 2078-2089.

[8] L. Lacasa, B. Luque, F. Ballesteros, J. Luque, and J.C. Nuño, Proc. Natl. Acad. Sci. USA., 105 (2008) $4972-4975$.

[9] B. Luque, L. Lacasa, F. Ballesteros, and J. Luque, Phys. Rev. E, 80 (2009) 046103.

[10] C.B. Li, H. Yang, and T. Komatsuzaki, Proc. Natl. Acad. Sci. USA., 105 (2008) 536-541.

[11] N. Marwan, J.F. Donges, Y. Zou, R.V. Donner, and J. Kurths, Phys. Lett. A, 373 (2009) 4246-4254.

[12] X.K. Xu, J. Zhang, and M. Small, Proc. Natl. Acad. Sci. USA., 105 (2008) 19601-19605.

[13] C. Liu and W.X. Zhou, J. Phys. A, 43 (2010) 495005.

[14] J.P. Eckmann, S.O. Kamphorst, and D. Ruelle, Europhys. Lett., 5 (1987) 973-977.

[15] J.P. Zbilut and C.L. Webber Jr., Phys. Lett. A, 171(3-4) (1992) 199-203.

[16] C.L. Webber Jr. and J.P. Zbilut, J. Appl. Physiol., 76(2) (1994) 965-973.

[17] TOCSY: http://tocsy.pik-potsdam.de

[18] J.P. Zbilut, A. Giuliani, and C.L. Webber Jr., Phys. Lett. A, 246(1-2) (1998) 122-128.

[19] N. Marwan and J. Kurths, Phys. Lett. A, 302(5-6) (2002) 299-307.

[20] M.C. Romano, M. Thiel, J. Kurths, and W.von Bloh, Phys. Lett. A, 330(3-4) (2004) 214-223.

[21] J.F. Donges, J.Heitzig, R.V. Donner, and J. Kurths, Phys. Rev. E, 85 (2012) 046105.

[22] R.V. Donner, J.F. Donges, Y. Zou, N. Marwan, and J. Kurths, In: Proceedings of the 2010 International Symposium on Nonlinear Theory and its Applications NOLTA2010 (Krakow 2010), Tokyo : IEICE 2010, p8790 . 
[23] B.B. Mandelbrot and J.W. Van Ness, SIAM Rev., 10(4) (1968) 422-437.

[24] L. Lacasa, B. Luque, J. Luque, and J.C. Nuño, Europhys. Lett., 86 (2009) 30001.

[25] W.J. Xie and W.X. Zhou, Physica A, 390 (2011) 3592-3601.

[26] Y. Zhou, Y. Leung, and Z.G. Yu, Phys. Rev. E, 87 (2013) 012921.

[27] M.A. Riley, R. Balasubramaniam and M.T. Turvey, Gait and Posture, 9 (1999) 65-78.

[28] J.S. Kim, K.I. Goh, G. Salvi, E. Oh, B. Kahng, and D. Kim, Phys. Rev. E, 75 (2007) 016110.

[29] B.G. Li, Z.G. Yu and Y. Zhou, J. Stat. Mech.: Theor. Exp., 2014 (2014) P02020.

[30] D.L. Wang, Z.G. Yu, and V. Anh, Chin. Phys. B, 21(8) (2012) 080504.

[31] N.H. Packard, J.P. Crutchfield, J.D. Farmer, and R.S. Shaw, Phys. Rev. Lett., 45(9) (1980) $712-716$.

[32] F. Takens, in: Rand D.A., Young L.S.(Eds), Dynamical Systems and Turbulence, Lecture Notes in Mathematics, Berlin: Springer-Verlag, 898 (1981) 366-381.

[33] M. Casdagli, S. Eubank, J.D. Farmer, and J. Gibson, Physica D, 51 (1991) 52-98.

[34] M.T. Rosenstein, J.J. Collins, and C.J. De Luca, Physica D, 73(1-2) (1994) 82-98.

[35] A.M. Fraser and H.L. Swinney, Phys. Rev. A, 33(2) (1986) 1134-1140.

[36] M.B. Kennel, R. Brown, and H.D. Abarbanel, Phys. Rev. A, 45(6) (1992) 3403-4311.

[37] L.Y. Cao, Physica D, 110(1-2) (1997) 43-50.

[38] N. Marwan, M.C. Romano, M. Thiel, and J. Kurths, Phys. Rep., 438(5-6) (2007) 237-329.

[39] M.A. Riley and G.C. Van Orden, Tutorials in contemporary nonlinear methods for the behavioral sciences (http://www.nsf.gov/sbe/bcs/pac/nmbs/nmbs.jsp) (2005).

[40] P. Erdős and A. Rényi, Publicationes Mathematicae Debrencen, 6 (1959) 290.

[41] M.S. Roulston, Physica D, 125(3-4) (1999) 285-294.

[42] Z.H. Tang and Z.G. Yu, Chin. J. Eng. Math., 29(4) (2012) 499-506.

[43] Gleich D.F., A graph library for Matlab based on the boost graph library, http://dgleich.github.com/matlab-bgl

[44] http://pajek.imfm.si/doku.php?id=pajek

[45] A.L. Barabási and E. Bonabeau, Scientific American, (2003) 50-59.

[46] X.H. Ni, Z.Q. Jiang, and W.X. Zhou, Phys. Lett. A, 373 (2009) 3822-3826.

[47] R. Milo, S.S. Orr, S. Itzkovitz, N. Kashtan, D. Chklovskii, and U. Alon, Science, 298 (2002) 824-827.

[48] R. Milo, S. Itzkovitz, N. Kashtan, R. Levitt, S.S. Orr, I. Ayzenshtat, M. Sheffer, and U. Alon, Science, 303 (2004) 1538-1542.

[49] http://www.weizmann.ac.il/mcb/UriAlon/

[50] B.B. Mandelbrot, Science, 155 (1967) 636-638.

[51] J. Feder, Fractals (Plenum, New York, 1988). 
[52] B.B. Mandelbrot, The Fractal Geometry of Nature (New York: Academic Press 1983).

[53] G. Palla, I. Derenyi, I. Farkas, and T. Vicsek, Nature, 435(7043) (2005) 814-818.

[54] K.I. Goh, G. Salvi, B. Kahng, and D. Kim, Phys. Rev. Lett., 96(1) (2006) 018701.

[55] C. Song, S. Havlin, and H.A. Makse, Nature, 433 (2005) 392-395.

[56] C. Song, L.K. Gallos, S. Havlin, and H.A. Makse, J. Stat. Mech.: Theor. Exp., 3 (2007) P03006.

[57] W.X. Zhou, Z.Q. Jiang, and D. Sornette, Physica A, 375(2) (2007) 741-752.

[58] R.W. Floyd, Commun. ACM, 5(6) (1962) 345.

[59] R.V. Donner, J. Heitzig, J.F. Donges, Y. Zou, N. Marwan, and J. Kurths, Eur. Phys. J. B, 84 (2011) $653-672$.

[60] E. Canessa, J. Phys. A: Math. Gen., 33 (2000) 3637-3651.

[61] V.V. Anh, Q.M. Tieng, and Y.K. Tse, Int. Trans. Oper. Res., 7 (2000) 349-363.

[62] Z.G. Yu, V. Anh, and K.S. Lau, Phys. Rev. E, 64 (2001) 031903.

[63] Z.G. Yu, V. Anh, and K.S. Lau, Phys. Rev. E, 68 (2003) 021913.

[64] Z.G. Yu, V. Anh, and K.S. Lau, J. Theor. Biol., 226 (2004) 341-348.

[65] Z.G. Yu, V. Anh, and K.S. Lau, Phys. Rev. E, 73 (2006) 031920.

[66] Z.G. Yu, V. Anh, and R. Eastes, J. Geophys. Res., 114 (2009) A05214.

[67] Z.G. Yu, V. Anh, Y. Wang, D. Mao, and J. Wanliss, J. Geophys. Res., 115 (2010) A10219.

[68] T.C. Halsey, M.H. Jensen, L.P. Kadanoff, I. Procaccia, and B.I. Shraiman, Phys. Rev. A, 33 (1986) $1141-1151$. 
Table 1: Comparison of recurrence networks transformed from FBM series with different Hurst indexes. Here the average is calculated from 100 realizations.

\begin{tabular}{c|c|c|c|c}
\hline$H$ & $\langle D(0)\rangle$ & $\langle D(1)\rangle$ & $\langle D(2)\rangle$ & $\Delta D(q)$ \\
\hline 0.40 & 1.632955359455532 & 1.462113762291203 & 1.324568844472558 & 1.675426636215924 \\
0.45 & 1.595331264113667 & 1.345243849453671 & 1.182356286524482 & 2.067323904428986 \\
0.50 & 1.570768526526059 & 1.294972986568832 & 1.135291034040322 & 2.132106915333559 \\
0.55 & 1.496955506340691 & 1.276092858298725 & 1.120419709203580 & 1.778923958082282 \\
0.60 & 1.471252546702877 & 1.264224152311605 & 1.090156202522773 & 1.740584602742986 \\
0.65 & 1.348210637236297 & 1.225915988658060 & 1.103150737927043 & 1.642556252215174 \\
0.70 & 1.304446382642433 & 1.134347097612430 & 0.989670372719278 & 1.488149695525952 \\
0.75 & 1.251720498742988 & 1.085148989401195 & 0.950681208097028 & 1.445178739248894 \\
0.80 & 1.206437282010590 & 1.064415121034396 & 0.949594824553587 & 1.375606925727599 \\
0.85 & 1.154054167326104 & 1.014055134463063 & 0.904518905840645 & 1.231289283108065 \\
0.90 & 1.106243766095298 & 0.974358969483122 & 0.869233664007547 & 1.084781493689348 \\
0.95 & 1.056322443543536 & 0.921690208443068 & 0.819310269771388 & 1.042386684931838 \\
\hline
\end{tabular}

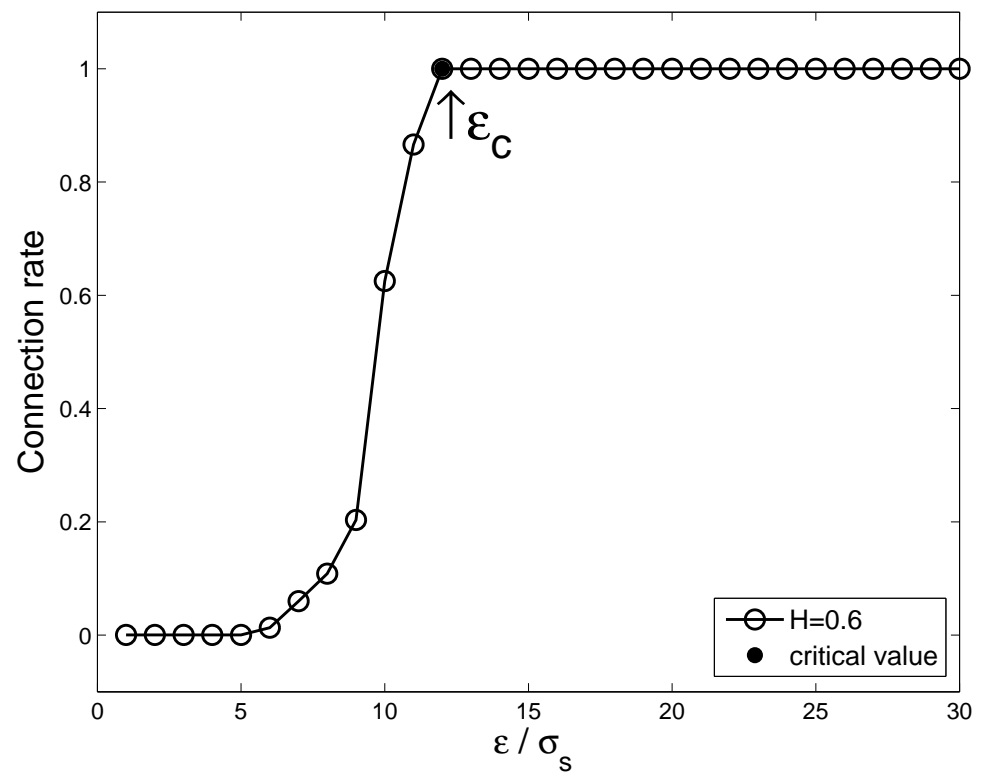

Figure 1: The relationship between the recurrence threshold $\varepsilon$ and the connection rate of the recurrence network constructed from fractional Brownian motion series of length $L=2^{12}$ with Hurst index $H=0.6$. 


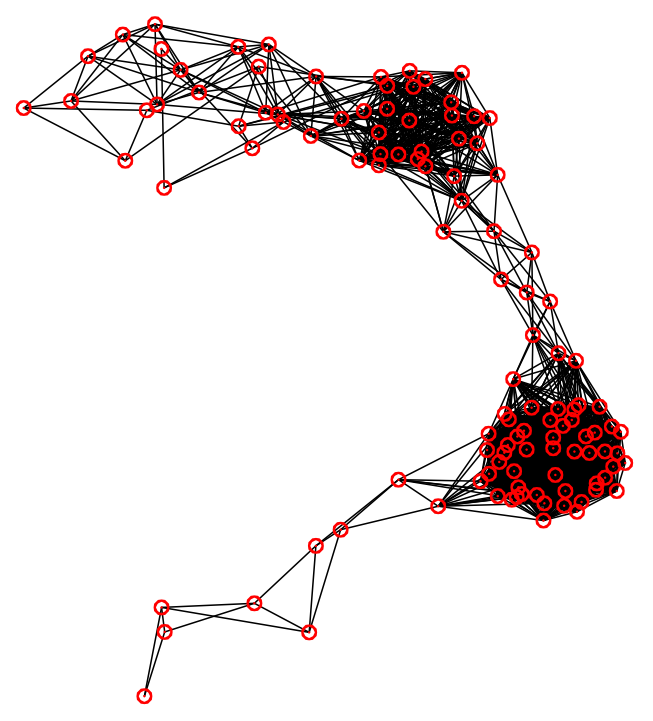

Figure 2: Recurrence network constructed from fractional Brownian motion series of length $L=2^{8}$ with Hurst index $H=0.6$.

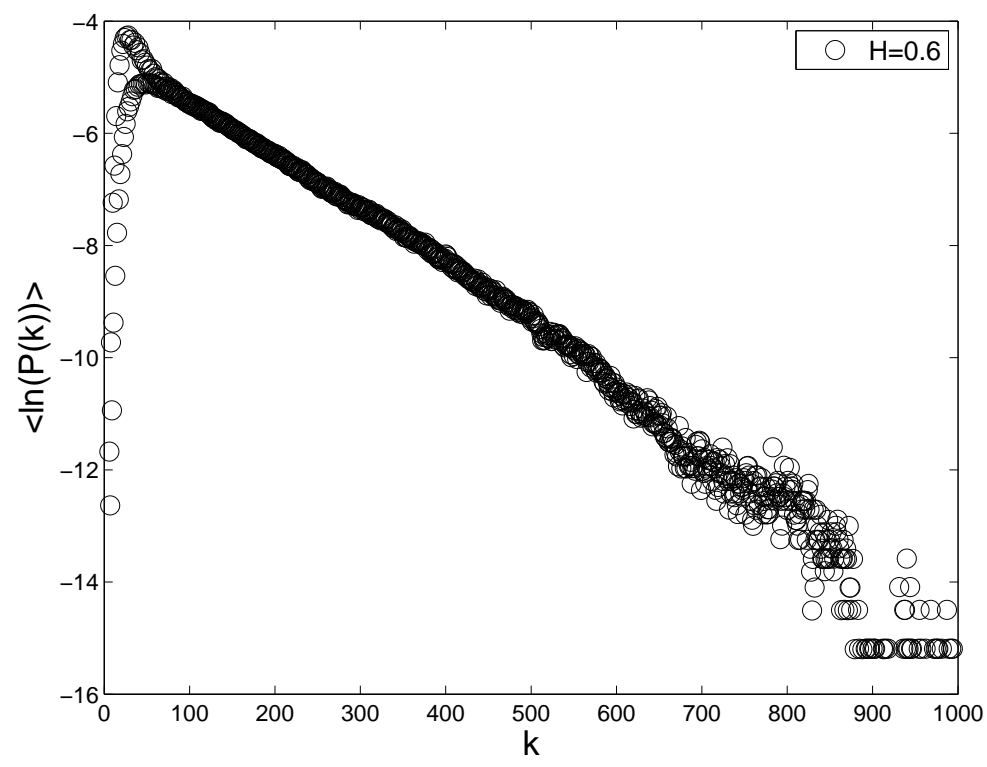

Figure 3: Degree distribution of one recurrence network constructed from fractional Brownian motion with Hurst index $H=0.6$. 


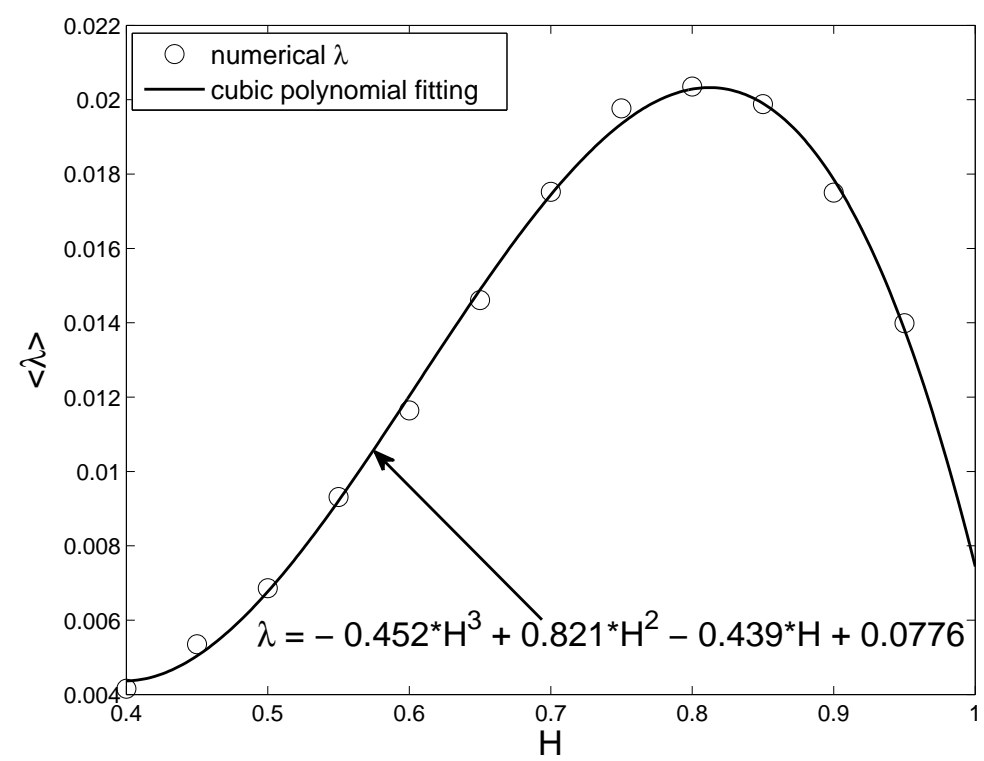

Figure 4: The relationship between $H$ of fractional Brownian motion and average degree exponent $\langle\lambda\rangle$ of the associated recurrence networks. Here the average is calculated from 1000 realizations.
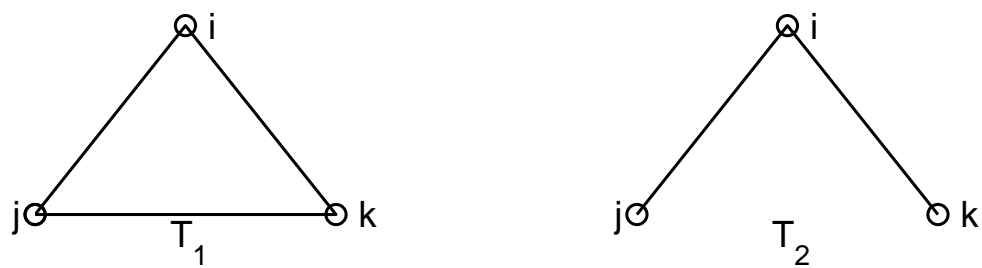

Figure 5: All two triples centered on vertex $i$.

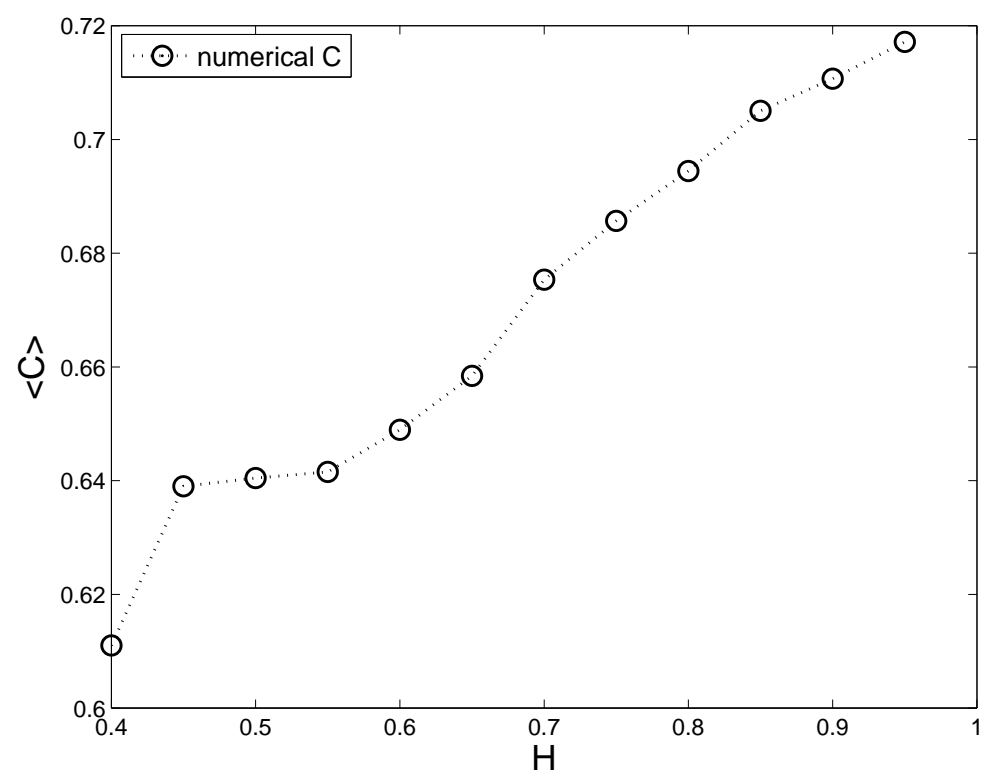

Figure 6: The relationship between $H$ of fractional Brownian motion and average clustering coefficient $\langle C\rangle$ of the associated recurrence networks. Here the average is calculated from 1000 realizations. 


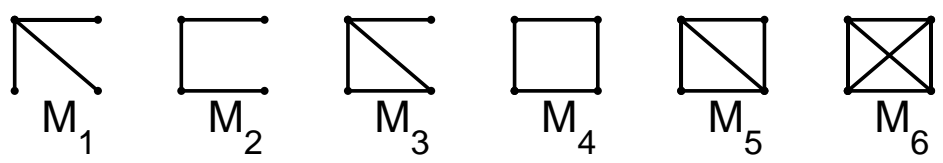

Figure 7: All six network motifs of size 4 in connected and undirected network. These motifs are labelled $M_{1}, M_{2}, M_{3}, M_{4}, M_{5}$, and $M_{6}$, respectively.
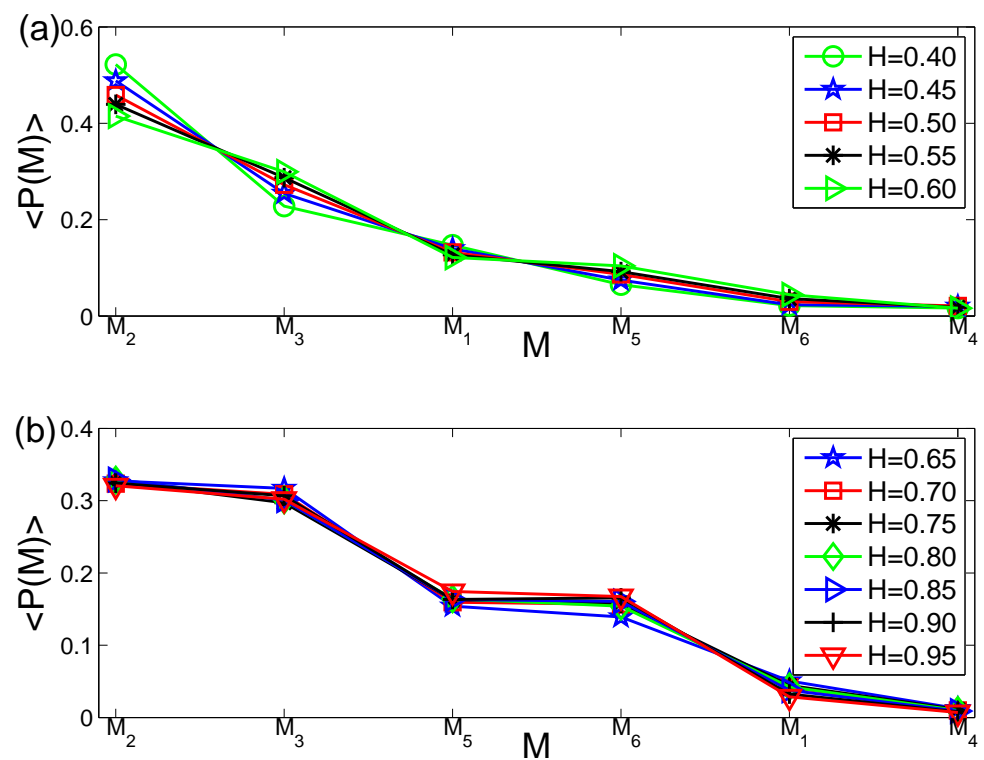

Figure 8: Network motif rank distributions of FBMs with different Hurst indices $H$. (a) Motif rank pattern $M_{2} M_{3} M_{1} M_{5} M_{6} M_{4}$ for $0.4 \leq H \leq 0.6$. (b) Motif rank pattern $M_{2} M_{3} M_{5} M_{6} M_{1} M_{4}$ for $0.65 \leq H \leq 0.95$. Here the average is calculated from 100 realizations.
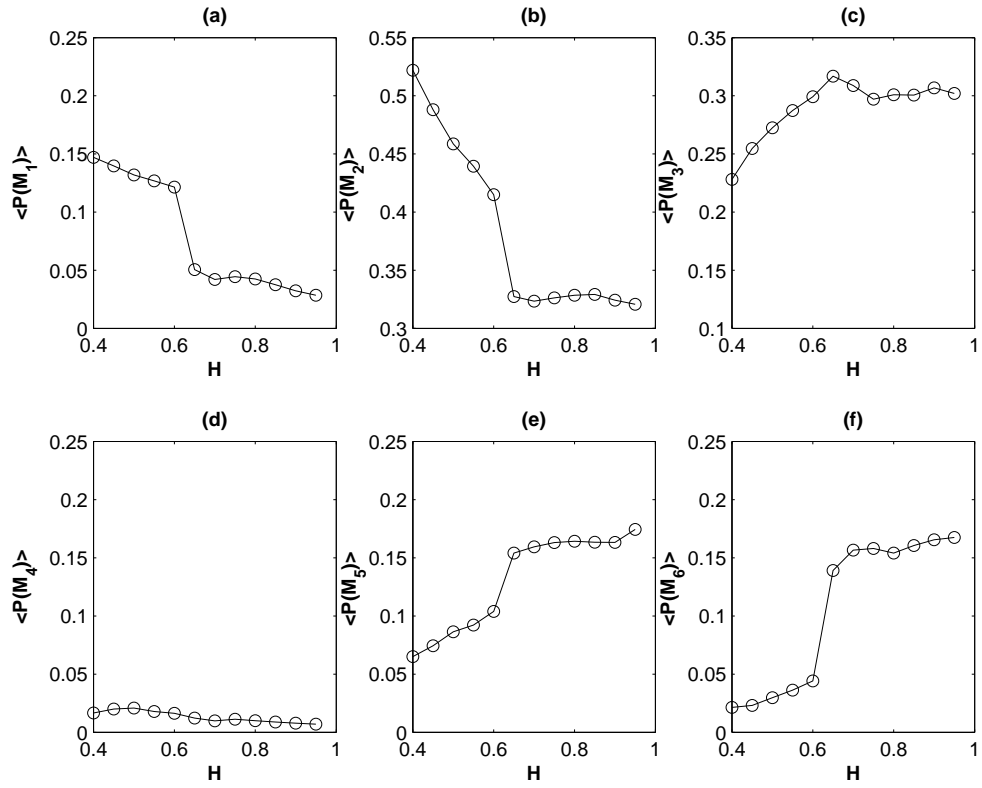

Figure 9: Dependence of average occurrence frequencies of motifs $M_{1}, M_{2}, M_{3}, M_{4}, M_{5}$, and $M_{6}$ on the Hurst indices $H$. Here the average is calculated from 100 realizations. 


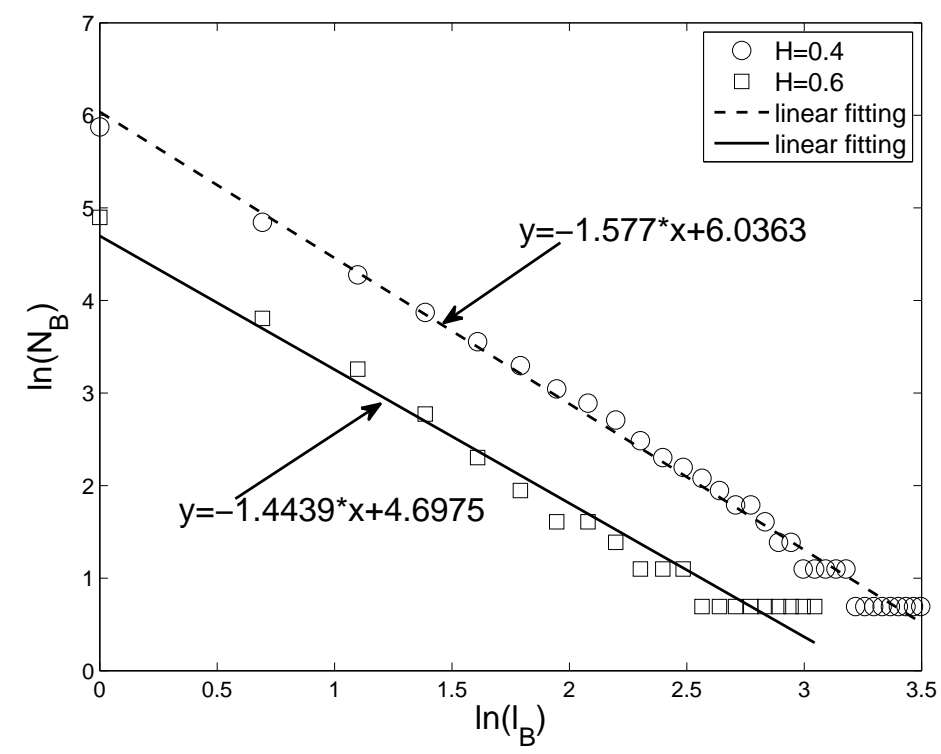

Figure 10: The fractal scaling of recurrence networks constructed from FBMs with Hurst indices $H=0.4$ and 0.6. The fractal dimension is the absolute value of the slope of the linear fit.

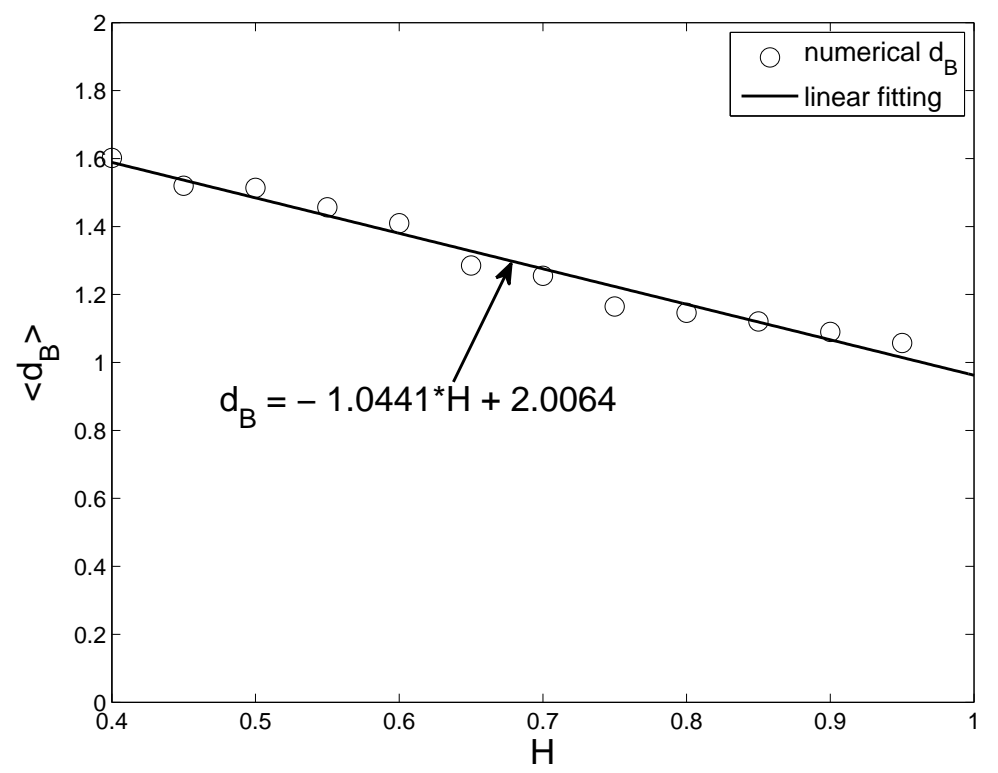

Figure 11: The relationship between $H$ of fractional Brownian motion and average fractal dimension $<d_{B}>$ of the associated recurrence networks. Here the average is calculated from 100 realizations. 


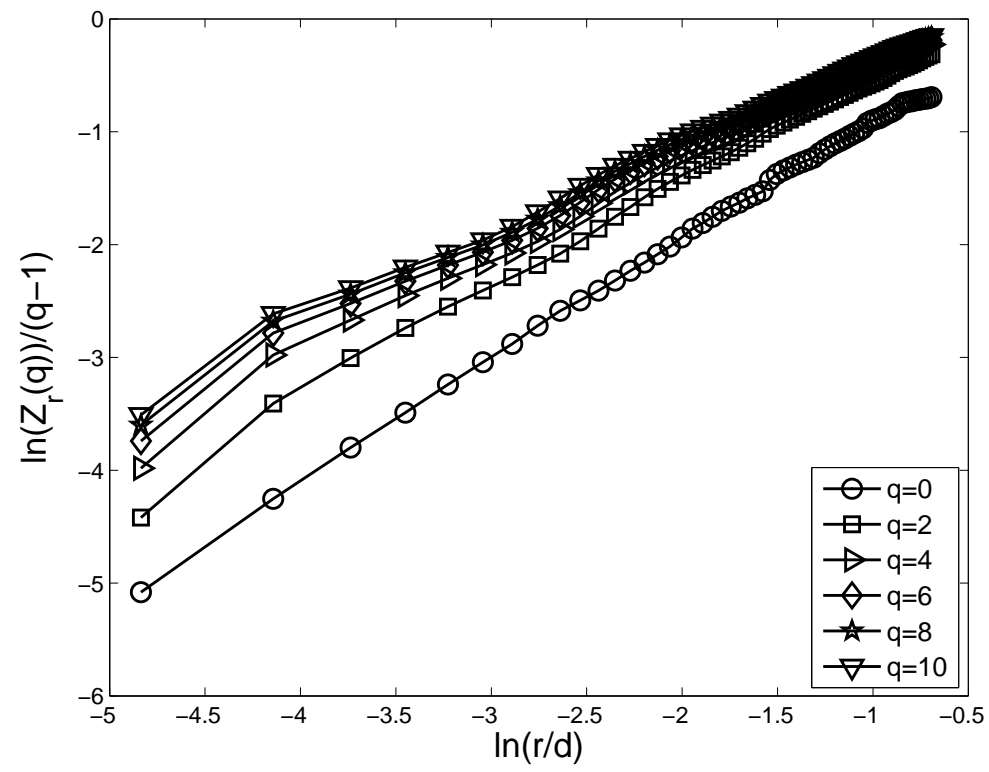

Figure 12: Linear regressions for calculating the generalized dimensions of the recurrence network.

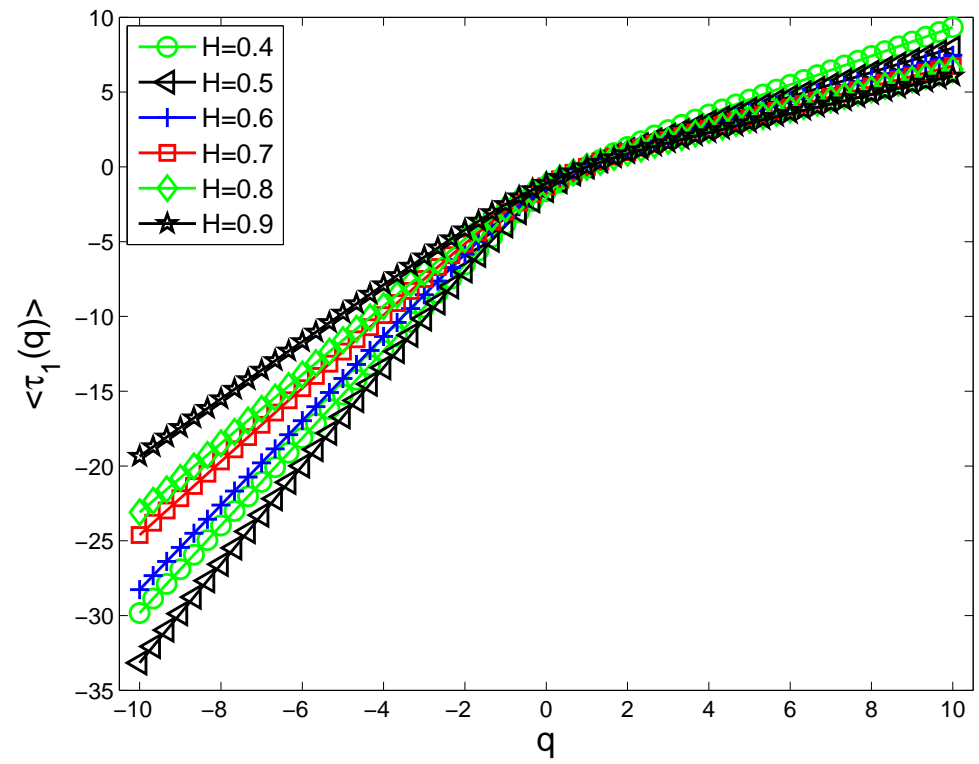

Figure 13: The average $\tau_{1}(q)$ curves of the recurrence networks. Here the average is calculated from 100 realizations. 


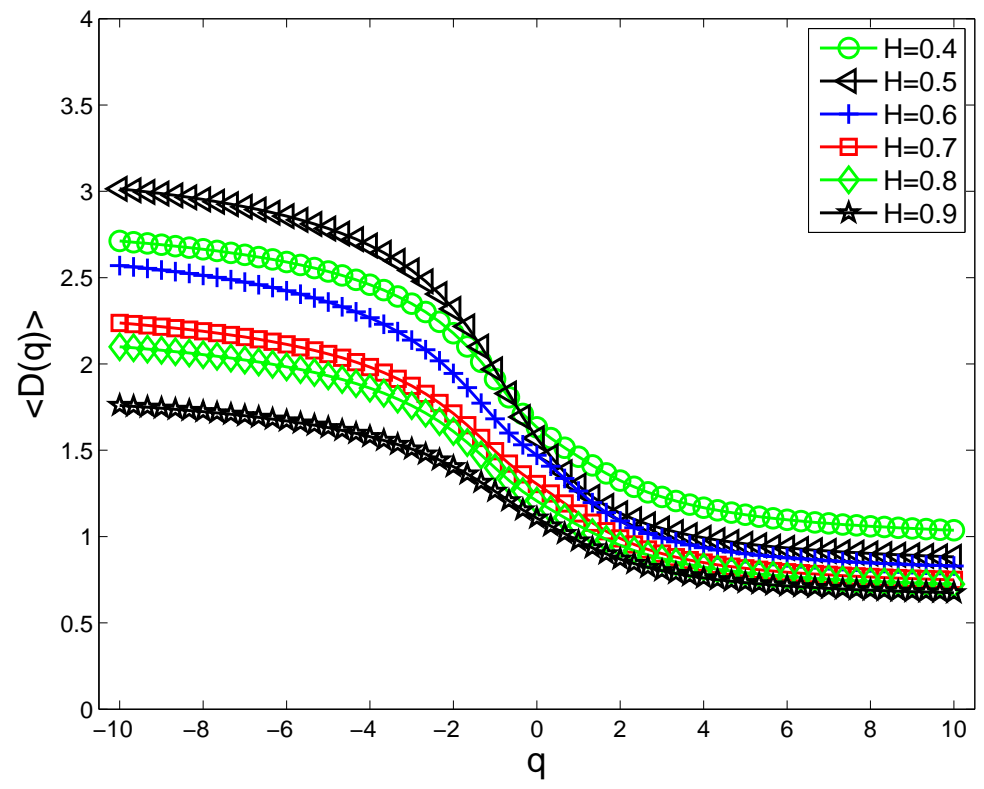

Figure 14: The average $D(q)$ curves of the recurrence networks, $D(0)$ is the fractal dimension of the network. Here the average is calculated from 100 realizations.
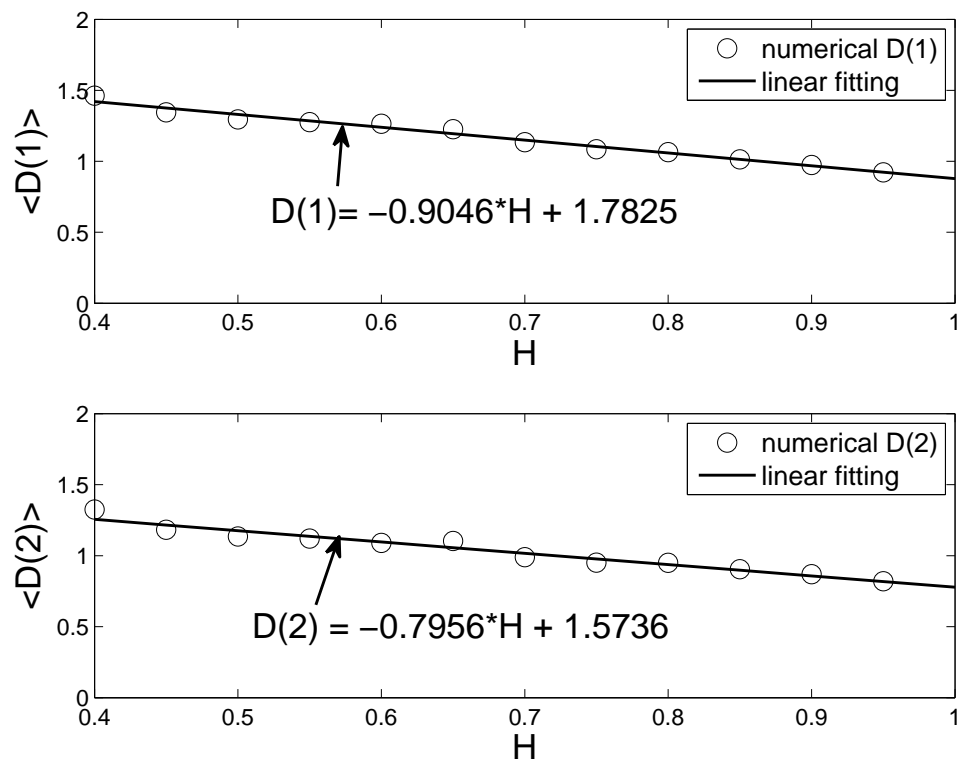

Figure 15: The dependence of the average information dimension $\langle D(1)>$ and the average correlation dimension $\langle D(2)>$ of the associated recurrence networks with respect to the Hurst index $H$ of fractional Brownian motion. Here the average is calculated from 100 realizations. 